\title{
Cyp26b1 Mediates Differential Neurogenicity in Axial-Specific Populations of Adult Spinal Cord Progenitor Cells
}

\author{
Carly Leung, ${ }^{1,2}$ Sherwin Chun Leung Chan, ${ }^{1}$ Sze Lan Tsang, ${ }^{1}$ Wutian Wu, ${ }^{2,3}$ and Mai Har Sham ${ }^{1,2}$
}

Utilization of endogenous adult spinal cord progenitor cells (SCPCs) for neuronal regeneration is a promising strategy for spinal cord repair. To mobilize endogenous SCPCs for injury repair, it is necessary to understand their intrinsic properties and to identify signaling factors that can stimulate their neurogenic potential. In this study, we demonstrate that adult mouse SCPCs express distinct combinatorial Hox genes and exhibit axialspecific stem cell properties. Lumbar-derived neurospheres displayed higher primary sphere formation and greater neurogenicity compared with cervical- and thoracic-derived neurospheres. To further understand the mechanisms governing neuronal differentiation of SCPCs from specific axial regions, we examined the neurogenic responses of adult SCPCs to retinoic acid (RA), an essential factor for adult neurogenesis. Although RA is a potent inducer of neuronal differentiation, we found that RA enhanced the generation of neurons specifically in cervical- but not lumbar-derived cells. We further demonstrate that the differential RA response was mediated by the RA-degrading enzyme cytochrome P450 oxidase b1 Cyp26b1. Lumbar cells express high levels of Cyp26b1 and low levels of the RA-synthesizing enzyme retinaldehyde dehydrogenase Raldh2, resulting in limited activation of the RA signaling pathway in these cells. In contrast, low Cyp26b1 expression in cervical spinal cord progenitor cells allows RA signaling to be readily activated upon RA treatment. The intrinsic heterogeneity and signaling factor regulation among adult SCPCs suggest that different niche factor regimens are required for sitespecific mobilization of endogenous SCPCs from distinct spatial regions of the spinal cord for injury repair.

\section{Introduction}

$\mathrm{N}$ EURAL STEM CELLS IN THE postnatal subventricular zone (SVZ) consist of diverse progenitor populations capable of giving rise to different neuronal subtypes depending on their position of origin [1]. However, the mechanisms that regulate this diversity remain unclear. Studies from neuronal fate specification within the embryonic spinal cord demonstrate that neuronal identity and diversification of neural progenitor cells is dependent on the position along the anteroposterior $(\mathrm{A} / \mathrm{P})$ and dorsoventral $(\mathrm{D} / \mathrm{V})$ axis [2]. A recent study in embryonic stem (ES) cells demonstrated that positional identity present in the ES-derived cell also determines subtype specification, and that altering the positional identity leads to corresponding changes in motor neuron subtype [3]. These studies suggest that the source of neural progenitor cells and its inherent positional code is a critical factor for generation of the desired neuronal subtypes. Whether all neural progenitor cells in the adult central nervous system (CNS) harbor such diversity and positional identity remains to be determined.
In the adult spinal cord, endogenous progenitor cells are distributed along the ependymal layer lining the central canal and from glial progenitors in the parenchyma of the spinal cord [4-6]. These cells can provide a source of repair during injury conditions. Genetic labeling studies using FoxJ1-Cre and Nestin-Cre mice pinpointed that spinal cord progenitor cells (SCPCs) originate from the ependymal layer cells surrounding the central canal [7]. In the intact spinal cord, SCPCs can self-renew or differentiate into astrocytes and oligodendrocytes, but rarely into neurons [4]. Under injury conditions, endogenous progenitor cells are induced to proliferate under the influence of cytokines and subsequently differentiate to astrocytes and oligodendrocytes $[8,9]$. Nevertheless, endogenous SCPCs rarely generate neurons in either the intact or injured spinal cord and appear to have limited contribution to regeneration after spinal cord injury [4,8-11]. However, while SCPCs are not neurogenic in their native niche, they can give rise to neurons in vitro when expanded with growth factors $[12,13]$ or when transplanted into the neurogenic hippocampus [14]. These studies demonstrate the multipotency of endogenous SCPCs, and that

${ }^{1}$ Department of Biochemistry, ${ }^{2}$ Centre for Reproduction, Development and Growth, and ${ }^{3}$ Department of Anatomy, Li Ka Shing Faculty of Medicine, The University of Hong Kong, Pokfulam, Hong Kong, China. 
environmental cues can influence their neurogenic potential. Despite several efforts [16], there is a lack of knowledge about the molecular mechanisms controlling neurogenesis of endogenous SCPCs.

During development, patterning of the spinal cord occurs under the influence of multiple transcriptional networks and inductive cues along the $\mathrm{D} / \mathrm{V}$ and $\mathrm{A} / \mathrm{P}$ axis. Retinoic acid (RA), in addition to being an inductive cue, is also essential for adult neurogenesis [15]. RA regulates multiple aspects of neurogenesis in the postnatal forebrain SVZ, including neural progenitor proliferation and migration of neuroblasts into the olfactory bulb [16], and is commonly used in in vitro differentiation protocols to generate neurons from ES cells and neural stem cells [16-18].

Since understanding the basic properties of endogenous SCPCs is key to their utilization for regenerative repair, we sought to define the cellular and molecular properties of SCPC subpopulations along the A/P axis. In this study, we show that there are distinct neural progenitor cell populations along the $\mathrm{A} / \mathrm{P}$ axis of the adult mouse spinal cord that display distinct positional identity conferred by specific $H o x$ gene signatures. Furthermore, we found that RA signaling led to an increase in neurogenesis in cervical- but not lumbar-derived progenitor cells. We found that this differential responsiveness was due to the high expression of the RA degradative enzyme Cyp26b1 in lumbar progenitor cells. Inhibition of Cyb26b1 in turn led to increased RA-induced neurogenesis. Our findings have important implications for devising novel strategies for manipulation of endogenous spinal cord progenitor cells in repair of spinal cord related disorders.

\section{Materials and Methods}

\section{Spinal cord progenitor cell isolation and culture}

Spinal cords of 6- to 8-week-old C57B6 mice (Laboratory Animal Unit, The University of Hong Kong) were used for SCPC isolation. All procedures on animals were approved by the Animal Ethics Committee of The University of Hong Kong. Spinal cord tissue was harvested from cervical (C1C6), mid-thoracic (T4-T9), and lumbar (L1-L6) regions, and progenitor cell isolation was performed as previously described [12]. Briefly, tissues were placed in artificial cerebrospinal fluid containing $124 \mathrm{mM} \mathrm{NaCl}, 5 \mathrm{mM} \mathrm{KCl}, 1.3 \mathrm{mM}$ $\mathrm{MgCl}_{2}, 2 \mathrm{mM} \mathrm{CaCl}_{2}, 26 \mathrm{mM} \mathrm{NaHCO}_{3}$, and $10 \mathrm{mM}$ D-glucose, $\mathrm{pH}$ 7.4. The tissue was cut into smaller pieces and digested in enzyme mixture containing $1.33 \mathrm{mg} / \mathrm{mL}$ trypsin, $0.67 \mathrm{mg} /$ $\mathrm{mL}$ hyaluronidase, and $0.2 \mathrm{mg} / \mathrm{mL}$ kynurenic acid (SigmaAldrich) for $30 \mathrm{~min}$ at $37^{\circ} \mathrm{C}$. Cells were dissociated mechanically and resuspended in Dulbecco's modified Eagle medium/F-12 medium (Invitrogen). Dissociated cell suspension was spun down, resuspended, and filtered through a $40-\mu \mathrm{m}$ nylon mesh (Becton Dickinson). The pellet was washed twice with phosphate buffered saline (PBS) and cultured as free-floating spheres at a plating density of 5,00010,000 cells $/ \mathrm{mL}$. Cells were cultured in standard neural stem cell (NSC) medium containing B27, basic fibroblast growth factor (bFGF), and epidermal growth factor (EGF) (20 ng/ $\mathrm{mL}$ ) (Invitrogen). Primary neurospheres were detected after 7-8 days in vitro. Growth factors were added twice per week. For neurosphere passaging, spheres were spun down, dissociated with mechanical trituration in $0.5 \times$ TrypLE (In- vitrogen), and cultured in NSC medium at 10,000 cells/mL for the generation of secondary spheres.

For differentiation experiments, secondary spheres were dissociated and resuspended in neurobasal medium (Invitrogen) supplemented with B27 and 5\% fetal bovine serum (FBS), without bFGF and EGF. Dissociated cells were plated onto poly-L-ornithine/laminin-coated (Sigma-Aldrich) LabTek 8-well chamber slides (Nalge-Nunc International) for 5 days. For experiments with retinoic acid, $1.0 \mu \mathrm{M}$ RA or dimethyl sulfoxide (DMSO) (Sigma-Aldrich) as control was added to neurobasal medium supplemented with B27 and without bFGF and EGF. In experiments assessing neuronal proliferation, $10 \mu \mathrm{M}$ 5'bromo-2'deoxyuridine (BrdU) was added to the differentiation medium.

For Cyp26b1 inhibition experiments, $1 \mu \mathrm{M}$ of talarozole (also known as R115866 [19], Hangzhou Onicon Chemical Co.) was added to the neurosphere culture 2 days prior to differentiation. Talarozole was freshly added each day in the presence or absence of $1 \mu \mathrm{M}$ RA during differentiation.

\section{Immunostaining}

Cells were fixed in freshly prepared $4 \%$ neutral buffered paraformaldehyde (PFA) for $15 \mathrm{~min}$ at room temperature. After blocking and permeabilization in PBS containing 5\% horse serum $/ 0.25 \%$ Triton-X100 for $30 \mathrm{~min}$, cells were incubated in antibodies recognizing $\beta$ III-tubulin (1:400, mouse monoclonal, Covance), glial fibrillary acidic protein (GFAP) (1:1,000, rabbit polyclonal, DAKO Corporation), O4 (1:500, mouse monoclonal, Chemicon), and BrdU (1:100, rat monoclonal, Abcam). For Cyb26b1 immunostaining, neurospheres were spun onto SuperFrost-Plus slides (Thermo Fisher Scientific) using Cytospin 2 cytocentrifuge (Shandon). After fixation and permeabilization as above, neurospheres were incubated with Cyp26b1 antibody (1:200, mouse monoclonal, Abnova). After PBS washes, samples were stained with secondary antibodies [1:400 Alexafluor 488 donkey anti-mouse immunoglobin G (IgG) conjugate or Alexafluor 594 donkey anti-rabbit IgG conjugate, Invitrogen]. For double staining with BrdU, the cells were first stained with primary and secondary antibodies. Subsequently, cells were treated with $2 \mathrm{~N} \mathrm{HCl}$ for $15 \mathrm{~min}$ at room temperature and incubated with anti-BrdU antibody. Subsequent to nuclear counterstaining with $4^{\prime} 6^{\prime}$ diamidino-2-phenylindole dihydrochloride (DAPI, 1:1,000, Sigma-Aldrich), samples were mounted with Fluromount-G (Southern Biotech).

\section{Quantitative analysis}

The number of differentiated cells was determined by counting the number of immunopositive cells as a percentage of total DAPI-positive nuclei in 10 random fields. Fluorescent cells were examined using Olympus BX51 fluorescent microscope.

\section{Reverse transcriptase polymerase chain reaction and quantitative polymerase chain reaction}

Secondary neurospheres were harvested for mRNA isolation using RNeasy RNA isolation kit (Qiagen). One microgram of RNA was reverse transcribed with oligo-dT and Superscript III reverse transcriptase (Invitrogen) according to manufacturer's protocol. The generated cDNA was used for semi-quantitative reverse transcriptase polymerase-chain 
A

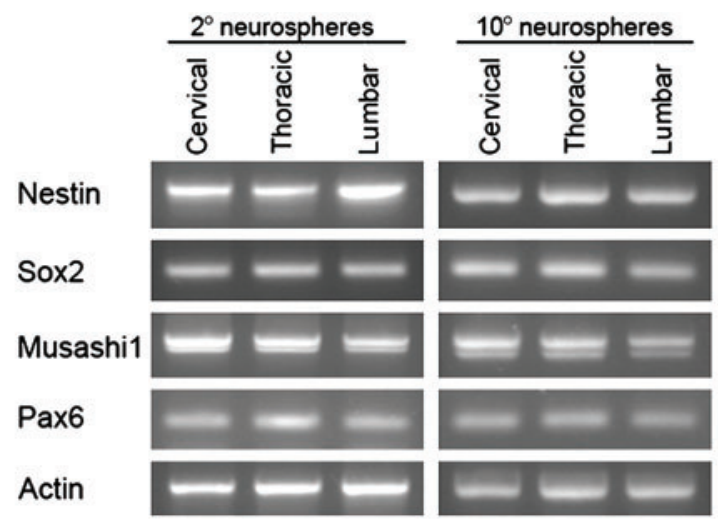

B

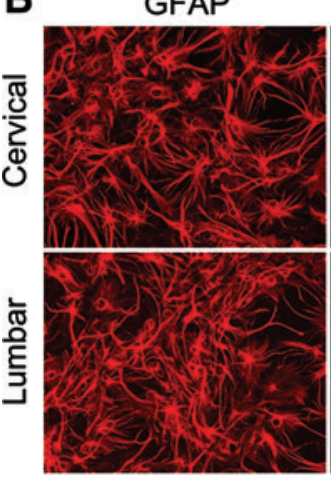

BIII-tubulin

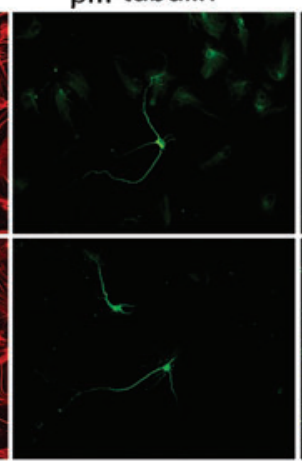

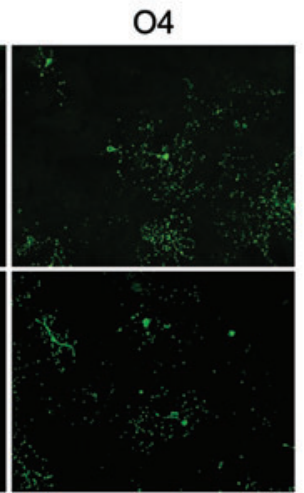

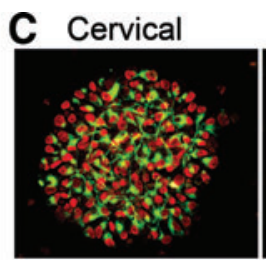

Thoracic
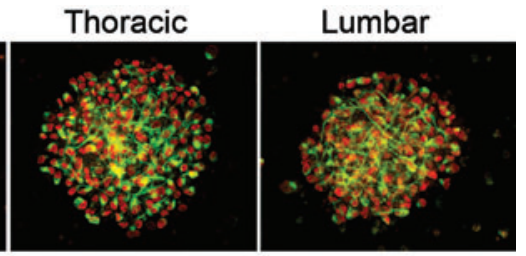

D

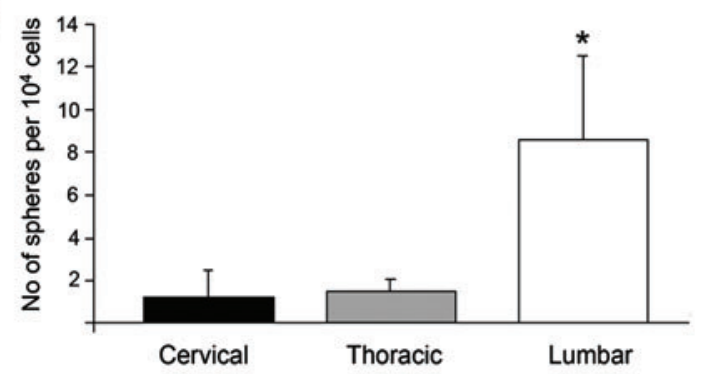

E

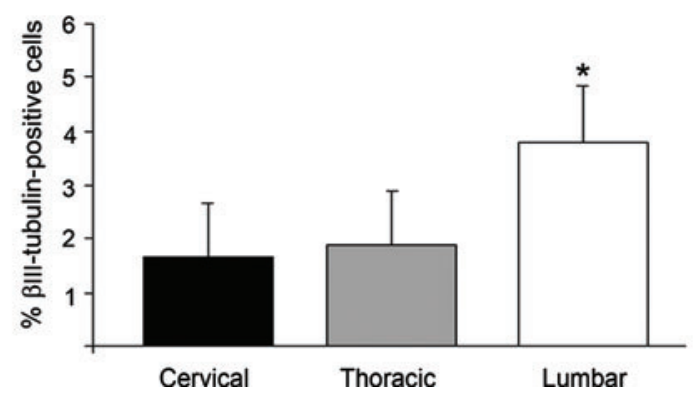

FIG. 1. Characterization of axial-derived spinal cord progenitor cells. (A) RT-PCR expression analysis of secondary neurospheres derived from cervical or lumbar regions of the adult spinal cord showing similar expression of Nestin, Sox2, Musashi-1, and Pax6. (B) Cervical and lumbar-derived spinal cord progenitor cells are multipotent. Representative immunofluorescent images of $\mathrm{O} 4$ (oligodendrocytes), GFAP (astrocytes) and $\beta$ III-tubulin (neurons) at 5 days of differentiation from cervical-derived (upper panel) and lumbar-derived (lower panel) secondary neurospheres (magnification, 200×). (C) Double immunofluorescent staining shows coexpression of of Nestin (green) and Sox2 (red) in neurospheres derived from cervical, thoracic and lumbar axial levels as indicated. (D) Primary sphere forming frequency for cervical, thoracic, and lumbarderived spinal cord progenitor cells. After 7 days of culture, higher number of neurospheres was observed from lumbar $(8.57 \pm 3.93)$ versus cervical $(1.20 \pm 1.27)$ and thoracic segments $(1.51 \pm 0.56) ;{ }^{*} P<0.05, n=4$. (E) Quantitation of $\beta I I I-t u b u l i n$ positive cells at 5 days of induced differentiation of dissociated neurospheres. Statistically significant higher percentages of immature neurons were observed in lumbar-derived spheres $(3.80 \pm 1.03 \%)$ versus cervical $(1.66 \pm 1.01 \%)$ and thoracic $(1.90 \pm 0.98 \%) ;{ }^{*} P<0.05, n=4$ (magnification, $\left.200 \times\right)$. Color images available online at www.liebertonline.com/scd

reaction (RT-PCR) using specific primers (Supplementary Table S1; Supplementary Data are available online at www.liebertonline.com/scd). PCR products were analyzed with $2 \%$ agarose gel.

For quantitative analysis, quantitative PCR (qPCR) was performed in triplicate with a StepOnePlus real-time PCR system (Applied Biosystems). PCR reaction mixtures containing cDNA template, specific primers, and Power SYBR Green (Applied Biosystems) in a final volume of $25 \mu \mathrm{L}$ was amplified with 40 cycles of $95^{\circ} \mathrm{C}$ for $15 \mathrm{~s}$ and $60^{\circ} \mathrm{C}$ for $60 \mathrm{~s}$. Melting curve analysis was performed to ensure the absence of non-specific products. Relative quantitation was carried out using the comparative CT method. A list of specific primers is provided in Supplementary Table 1.

\section{Statistical analysis}

All data are represented as mean \pm standard error of the mean and were analyzed using paired Student's $t$-test. $P$ values $<0.05$ were considered to be statistically significant.

\section{Results}

\section{Adult spinal cord progenitor cells along the $A / P$ axis have distinct cellular properties}

To determine the properties of neural progenitor cell populations along the $\mathrm{A} / \mathrm{P}$ axis of the adult spinal cord, we isolated progenitor cells from 3 anatomical regions of the mouse spinal cord: cervical (C3-C7), mid-thoracic (T4-T9), and lumbosacral (L1-L6). We examined their neural progenitor cell characteristics first by expression analysis of neural stem cell markers performed on neurospheres derived from each of the 3 anatomic regions. Each of the 3 SCPC populations expressed genes associated with adult neural progenitor cells-Nestin, Sox2, Musashi-1, and Pax6-to a similar degree (Fig. 1A, left). This expression pattern was maintained through to the 10th passage (which was the duration of our experiment), indicating a maintenance of neural stem cell character through multiple rounds of cell division (Fig. 1A, right). To examine their cell properties in vitro, we assessed neurosphere formation ability, 
self-renewal capacity, and differentiation potential. Cells in the neurosphere were positive for Sox 2 and Nestin (Fig. 1C). We found that significantly more neurospheres were derived from the lumbar region compared with cervical and thoracic regions $(8.58 \pm 3.93$ in lumbar, $1.20 \pm 1.27$ in cervical, and $1.51 \pm 0.56$ in thoracic regions; $P<0.05, n=4$ ) (Fig. 1D). This suggests that a higher number of progenitor cells were present in the lumbar region. Primary spheres from each of the 3 regions did not appear to differ in their ability to form secondary spheres (data not shown).

We assessed the 3 SCPC populations for their differentiation capacity under spontaneous differentiation conditions without bFGF and EGF and in the presence of 5\% FBS. Neural progenitor cells derived from each of the 3 regions were multipotent, capable of giving rise to differentiated $\beta \mathrm{III}-$ tubulin + neurons, GFAP + astrocytes, and O4+ oligodendrocytes (Fig. 1B), with the neurosphere cultures differentiating predominantly into astrocytes. However, though the cells were multipotent, SCPCs from different regions exhibited differences in neurogenicity (Fig. 1E). We observed a 2-fold higher number of neurons derived from lumbosacral-derived progenitor cells compared with cervical or thoracic-derived cells $(3.8 \% \pm 1.03$ in lumbar, $1.66 \% \pm 1.01$ in cervical, and $1.9 \% \pm 0.98$ in thoracic; $P<0.05, n=4)$, indicating differences in neuronal commitment between the cell populations. Our results indicate that progenitor cell populations isolated from different spatial regions of the spinal cord exhibit differences in progenitor cell properties.

\section{Adult SCPCs retain positional identity}

During development, the $\mathrm{A} / \mathrm{P}$ axis of the spinal cord is encoded by the expression of combinatorial Hox genes, which determines the positional identity and consequently the patterning and neuronal subtype specification of cells within the spinal cord tissue. In order to determine whether adult SCPCs along the length of the spinal cord preserved $\mathrm{A} / \mathrm{P}$ identity, we examined region-specific Hox gene expression profile by semi-quantitative RT-PCR. Secondary neurospheres derived from cervical, mid-thoracic, and lumbar regions were examined for their expression of regionspecific Hox genes (Fig. 2, left). We found that neurospheres from each anatomical region coexpressed a distinct combination of Hox genes, and successively more $5^{\prime}$ genes within a cluster were expressed more posteriorly. All 3 regions expressed Hoxc4, consistent with its expression in discrete segments of the neural tube [20]. Cervical progenitor cells expressed the anterior Hox genes Hoxc5 and Hoxc6 at higher levels, with low expression of the posterior 5' Hox gene Hoxc10. Cervical and thoracic neurospheres did not express the posterior genes Hoxa11, Hoxc11, and Hoxc13. Thoracic progenitors had the highest expression of Hoxc8 and Hoxc9, genes associated with thoracic identity. Lumbar progenitor cells expressed high levels of Hox genes from the paralogous groups 11 and 13, and relatively lower levels of the anterior Hoxc5 and Hoxc6 genes. This pattern of expression corresponds well to the notion that $3^{\prime}$ Hox paralogous groups are expressed by anterior cells, and 5' Hox paralogous groups are expressed by posterior-derived cells in the spinal cord. Therefore, a distinct combinatorial Hox code corresponding to the spatial region of the spinal cord persists in the adultderived spinal cord progenitor cells.

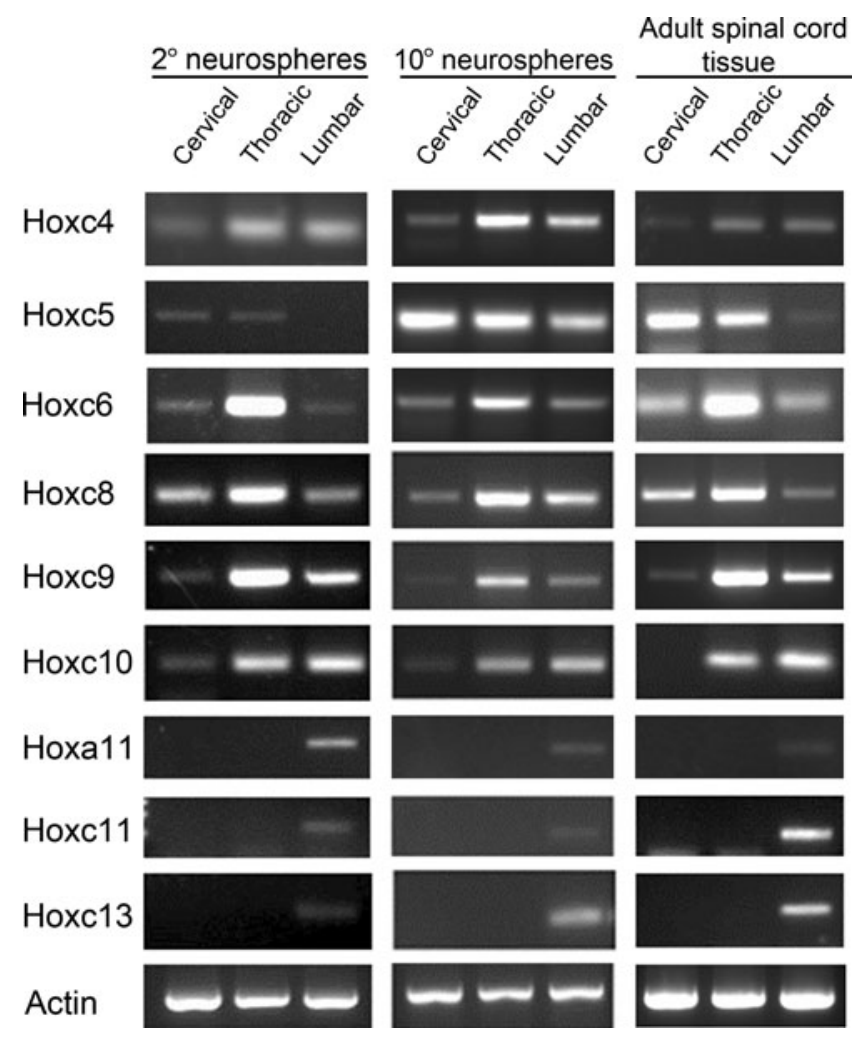

FIG. 2. Spinal cord progenitor cells retain positional identity. (Left) Semi-quantitative RT-PCR showing that cervical, thoracic, and lumbar-derived secondary neurospheres differentially expressed various Hox genes as indicated. $\beta$-actin expression was used as loading control. (Center) RT-PCR analysis of neurospheres shows that differential Hox gene expression is maintained after the 10th passage. (Right) Hox gene expression in cervical-, thoracic-, and lumbar-derived neurospheres mimics the expression observed in corresponding regions of the adult spinal cord tissue.

In order to determine whether the Hox signature expressed by SCPCs is maintained during prolonged culture conditions, we examined the expression of selected Hox genes in neurospheres that had been serially passaged at clonal density. We found that the expression of combinatorial Hox genes was unchanged through to the 10th passage (Fig. 2, center), indicating that Hox gene expression was not altered by long-term culture conditions, and that the anteroposterior positional identity is stably maintained through multiple rounds of cell division.

Although the expression of Hox code is well established during embryonic development, it remains unclear whether this gene signature is preserved in the adult spinal cord tissue from which the progenitor cells were derived. To determine whether the Hox gene signature observed in the various spinal cord populations reflected the expression pattern in the adult, we examined the expression of selected Hox genes in adult spinal cord homogenates (Fig. 2, right). We found that the adult cervical, thoracic and lumbosacral spinal cord also exhibited a distinct Hox gene expression pattern, and that this Hox expression profile was faithfully recapitulated in the neural progenitor cells derived from each region. 


\section{Retinoic acid enhances neurogenesis in cervical-derived but not lumbar SCPCs}

Since neurogenesis from SCPCs is difficult to achieve in vivo, we asked whether neurogenesis could be enhanced using niche factors. Retinoic acid is a well-established agent for inducing neurogenesis from ES cells and brain-derived NSCs $[16,17]$, and therefore could potentially be effective in promoting neurogenesis in SCPCs. To this end, secondary neurospheres from cervical and lumbar regions were dissociated and differentiated in the presence of 1.0uM RA for 5 days, and neurons were immunostained with $\beta$ III-tubulin (Fig. 3C). By quantitative analysis, we found that RA significantly increased the number of $\beta$ III-tubulin-positive neurons by approximately 2-fold (2.31 $\pm 0.59, P<0.05, n=3$; Fig $3 \mathrm{~A})$ in cervical-derived SCPC populations. Surprisingly, no change in neuronal number in lumbar-derived progenitor cells was observed.

To determine whether RA-induced neurogenesis in cervical populations occurred through changes in neuronal cell commitment, or increase in neural progenitor cell proliferation or survival, we exposed cervical and lumbar SCPC cultures to BrdU throughout the culture period. We found that the number of BrdU and $\beta$ III-tubulin double-immunopositive neurons was increased by 2 -fold in cervical SCPCs $(12.05 \pm 2.7$ vs. $5.17 \pm 1.8$ for control; Fig. $3 \mathrm{~B}$ ), accounting for the increase in neuronal differentiation observed. The total number of BrdU-positive cells remained the same in RA-treated cervical SCPC cultures, indicating that overall proliferation was not affected. Altogether, we demonstrate that RA enhances neuronal proliferation in cervical-derived SCPCs while lumbarderived cells remained unresponsive.

\section{$R A$ induces upregulation of anterior Hox genes, but overall Hox gene signature is unaffected}

In addition to stimulating neurogenesis, RA acts as a positional cue for the patterning of the neural tube through the regulation of Hox genes. Therefore, we asked whether the neurogenic effects of RA might be due to changes in Hox gene signature of spinal cord progenitor cells. We examined the Hox gene signature using $\mathrm{qPCR}$ in cervical or lumbar neurospheres after treatment with $1.0 \mu \mathrm{M}$ RA (Fig. 4). While HoxA genes remained unchanged in either cervical or lumbar SCPCs, we found that the anterior Hoxc4 gene expression was increased by approximately 5-fold in cervical SCPCs after 1 day, but relatively unchanged in lumbar SCPCs. Hoxc5 expression was increased in both cervical and lumbar populations. Hoxc6, c8, $c 9, c 10, c 11$, and $c 13$ gene expression were unchanged in both cervical and lumbar populations. That is, the posterior genes Hoxa11, c11, and c13 remained absent in cervical SCPCs after RA treatment, indicating that cervical identity was maintained. This indicated that although retinoic acid could induce specific Hox gene upregulation, the overall Hox gene signature of the axial populations remained unchanged, suggesting that other mechanisms could be responsible for the lack of RA-induced neurogenesis observed in lumbar SCPCs.

\section{Differential Cyp26b1 expression in SCPCs determines sensitivity to RA signaling}

In order to determine the molecular basis for the differential response to RA observed in the different axial-derived
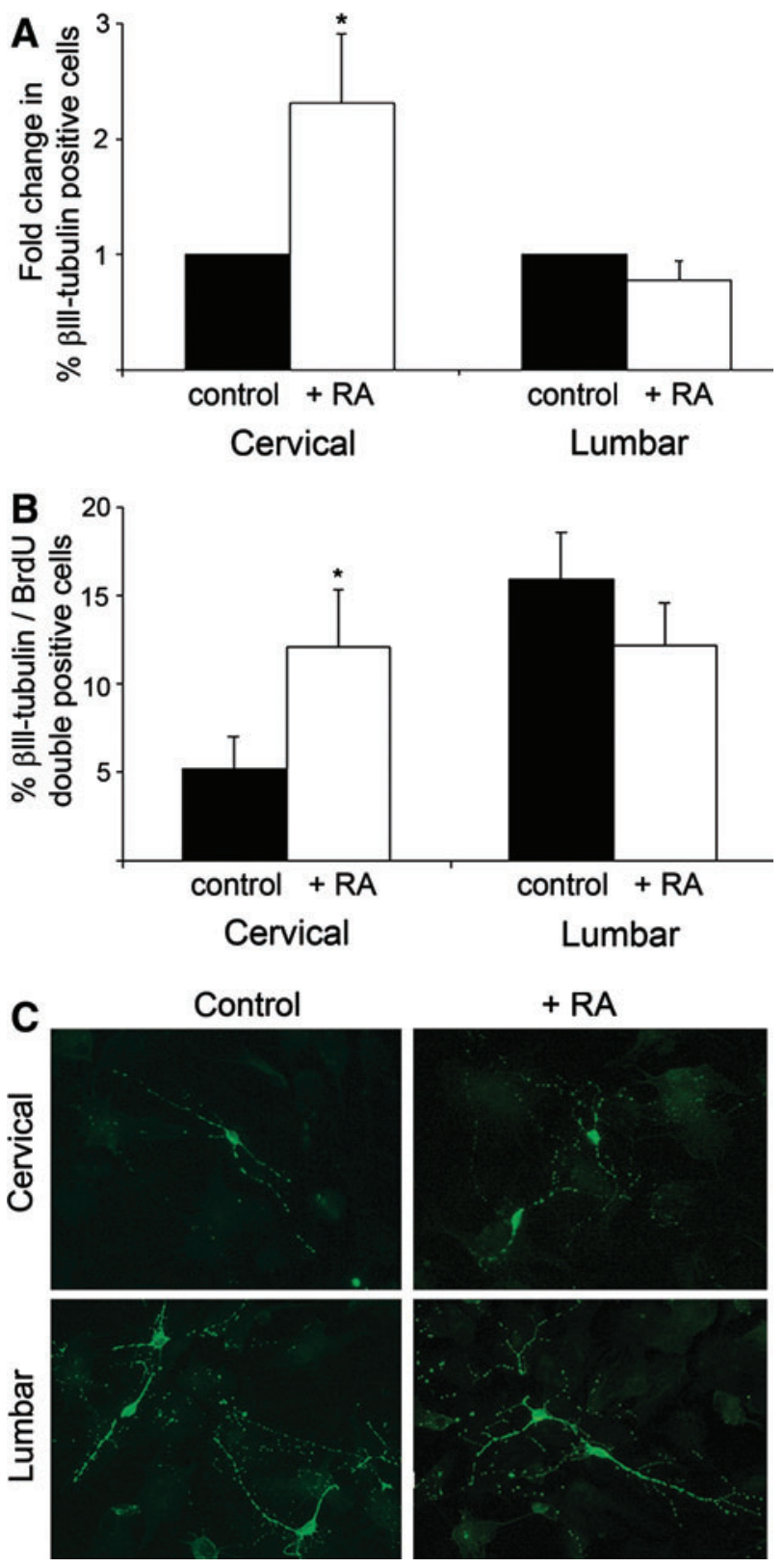

FIG. 3. Axial-derived spinal cord progenitor cells (SCPCs) respond differently to retinoic acid RA treatment. (A) Neurogenicity is enhanced in cervical but not lumbar-derived cultures after differentiation in the presence of $1.0 \mu \mathrm{M}$ RA for 5 days. Bars represent the fold change in increase of $\beta$ III-tubulin cells after treatment with $1.0 \mu \mathrm{M}$ RA (black bar) versus untreated control (white bar) which is normalized to 1. Approximately 2-fold increase in RA-induced neurogenesis was observed for cervical SCPCs compared to untreated cervical cells $\left(2.31 \pm 0.59\right.$-fold; $\left.{ }^{*} P<0.05\right)$, whereas no difference in lumbar neurogenesis was observed $(0.78 \pm 0.17$-fold). Bars represent mean \pm standard error of the mean (SEM) from at least 3 independent experiments. (B) The percentage of $\beta I I I-$ tubulin/BrdU double-positive cells increased significantly in cervical-derived SCPC differentiation 5 days after RA treatment $\left(12.05 \pm 2.7\right.$ vs. $5.17 \pm 1.8 \%$ for untreated control; ${ }^{*} P<0.05$, $n=3)$. (C) Immunofluorescence of $\beta I I I-t u b u l i n$ showing SCPCdifferentiated neurons from cervical and lumbar segments with or without RA induction (magnification, 200×). Color images available online at www.liebertonline.com/scd 


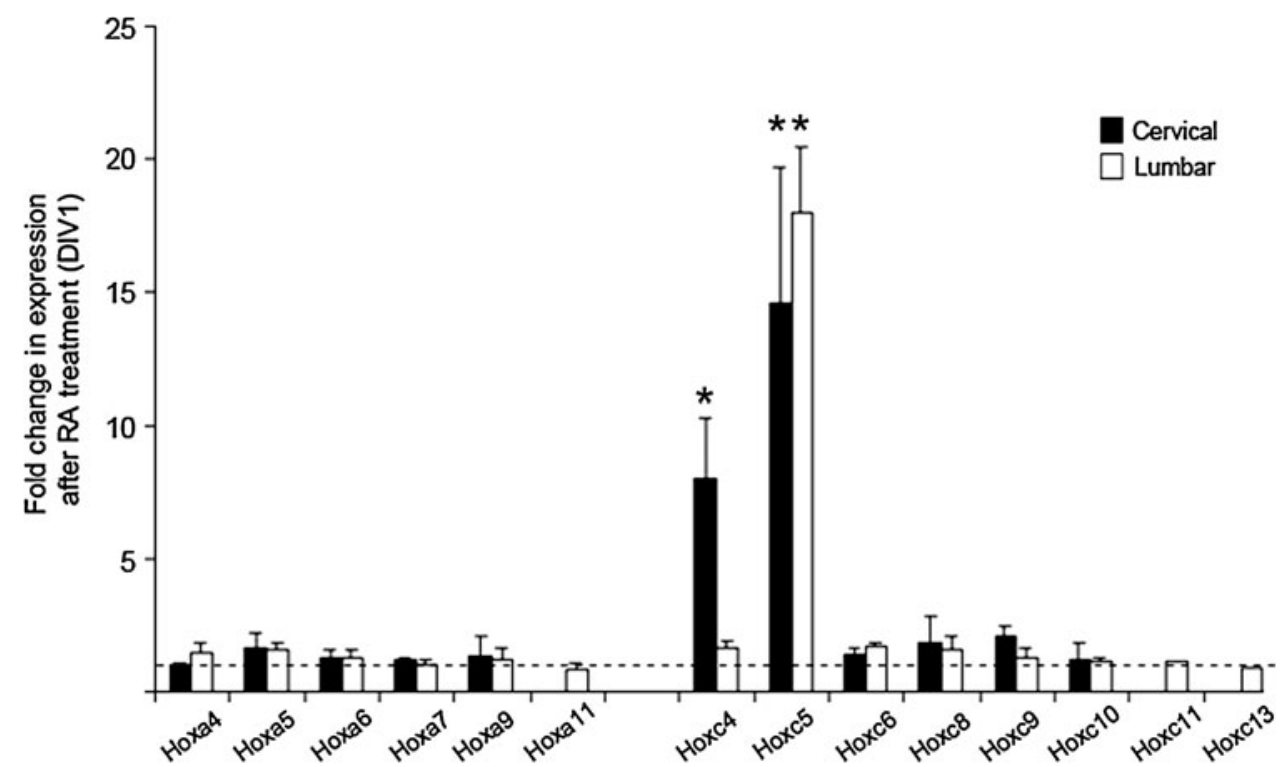

FIG. 4. Retinoic acid treatment induces changes in Hox gene expression. Relative fold changes in Hox gene expression in cervical-derived (black bars) and lumbar- (white bars) derived neurospheres at 1 day of treatment with $1 \mu \mathrm{M}$ RA as analyzed by qPCR. Each bar (mean \pm SEM) represents data normalized to DMSO-treated controls of the corresponding cell populations. Statistically significant higher expression of Hoxc4 $\left(7.98 \pm 2.29\right.$-fold) compared to untreated control $\left({ }^{*} P<0.05\right)$ was observed for cervical cell populations, and Hoxc5 for both cervical (14.61 \pm 5.08 -fold) and lumbar (17.96 \pm 2.43 -fold) populations after $1.0 \mu \mathrm{M}$ RA treatment. Dotted line is set at the value of 1 . All data were normalized to GAPDH control. Bars represent means of at least 3 independent experiments $\left({ }^{*} P<0.05, n=3\right)$.

SCPCs, we examined the expression of various retinoidsignaling components including RA receptors and metabolic enzymes by quantitative RT-PCR. Cervical and lumbar progenitor cell populations both expressed receptors associated with RA signaling, RAR-alpha, beta, and gamma and RXR-alpha, beta, and gamma to a similar degree (Fig. 5A). However, compared to lumbar-derived spheres, we found a 4-fold lower expression of the P450 cytochrome oxidase 26b1 Cyp26b1, an enzyme which degrades RA into polar derivatives, in cervical spheres. Also, cervical cells had a 2- to 3-fold higher expression of the gene Raldh2, an enzyme involved in the conversion of retinaldehyde to RA. We also observed strong Cyp26b1 expression in lumbar spheres by immunostaining analysis (Fig. 5B), while expression was absent in cervical-derived spheres. Our results suggest that Cyp26b1 is a determining factor in regulating RA signaling activation and neurogenesis in SCPCs.

\section{Enhanced RA-induced neurogenic response in lumbar cells in the presence of Cyp26b1 inhibitor}

In order to determine whether Cyp26b1 is indeed responsible for the lack of RA-mediated neurogenic response, we treated cells with the pharmacological agent talarozole (formerly R11866), a Cyp26b1 inhibitor that results in increased RA signaling in vivo [19]. The inhibitor was added to neurosphere cultures 2 days prior to neuronal differentiation to ensure minimal Cyp26b1 activity during differentiation. Cervical and lumbar cells were treated with $1 \mu \mathrm{M}$ of talarozole for the entire experiment, cultured in the presence or absence of $1 \mu \mathrm{M}$ RA during differentiation, followed by immunostaining and quantification of the number of $\beta I I I-$ tubulin neuronal cells. In lumbar-derived cultures, we ob- served an approximately 1.5 -fold increase in the number of $\beta$ III-tubulin-positive neurons in RA-treated lumbar cultures in the presence of talarozole compared with control cells $(P<0.05)$, indicating RA pathway activation in these cells. Our results demonstrate that RA-induced neurogenicity can be enhanced in lumbar-derived progenitor cells when Cyp26b1 activity is inhibited.

\section{Discussion}

Our study demonstrates that spinal cord progenitor cell populations along the anteroposterior length of the adult spinal cord are spatially defined populations characterized by distinctive regional-specific Hox profiles, indicating that regional specification is maintained from development to adulthood. Furthermore, we identified differential sensitivity to RA-enhanced neurogenesis among $\mathrm{A} / \mathrm{P}$ progenitor populations, which was regulated by the RA metabolizing enzyme Cyp26b1. Our findings have important implications for utilizing signaling molecules to mobilize endogenous spinal cord progenitor cells in regenerative therapy.

We demonstrate here that the spatially diverse populations in the adult spinal cord display varying cellular and molecular properties. A higher neurosphere frequency was observed for cells derived from the lumbar region, suggesting more abundant progenitor cells in this region. Consistent with previous reports, in cells derived from the adult rat spinal cord [6], lumbar-derived neurospheres had a higher propensity to differentiate toward neurons during spontaneous differentiation. It remains to be determined whether differences in behavior between anterior and posterior progenitor populations are due to the intrinsic property of 
FIG. 5. Differential expression of RA signaling genes in secondary neurospheres derived from axial spinal cord progenitor cells. (A) Quantitative real-time PCR analysis shows differential gene expression of raldh2 (2.42 \pm 0.32 -fold) and cyp26b1 (0.27 \pm 0.15 -fold) of cervical-derived neurospheres compared with lumbar neurospheres. Data represent fold difference in expression of cervical cell populations as normalized to lumbar cell expression of the corresponding genes $\quad\left({ }^{*} P<0.05, \quad n=3\right)$. (B) Cyp26b1 immunofluorescence staining of secondary spheres derived from cervical and lumbar progenitor cells. Cytoplasmic Cyp26b1 staining is apparent in lumbar-derived neurospheres, whereas no expression is observed in cervicalderived neurospheres. (C) RA significantly increases neuronal differentiation in lumbar progenitor cells after treatment with $1 \mu \mathrm{M}$ talarozole. Bars represent fold change in the number of $\beta I I I-t u b u l i n$ cells in 1.0- $\mu \mathrm{M}$ RA-treated cultures compared with control cultures not treated with RA. Compared with lumbar cultures without talarozole treatment, RA induced a mild increase in the number of $\beta$ III-tubulin-positive cells upon addition with $1 \mu \mathrm{M}$ talarozole $(1.48 \pm 0.23$ vs. $1.07 \pm$ 0.05 -fold change; $* P<0.05$, $n=3)$. No significant differences in the fold-change of $\beta$ IIItubulin cells in RA-induced cultures were found in talarozole-treated versus untreated cervical cultures. Color images available online at www .liebertonline.com/scd

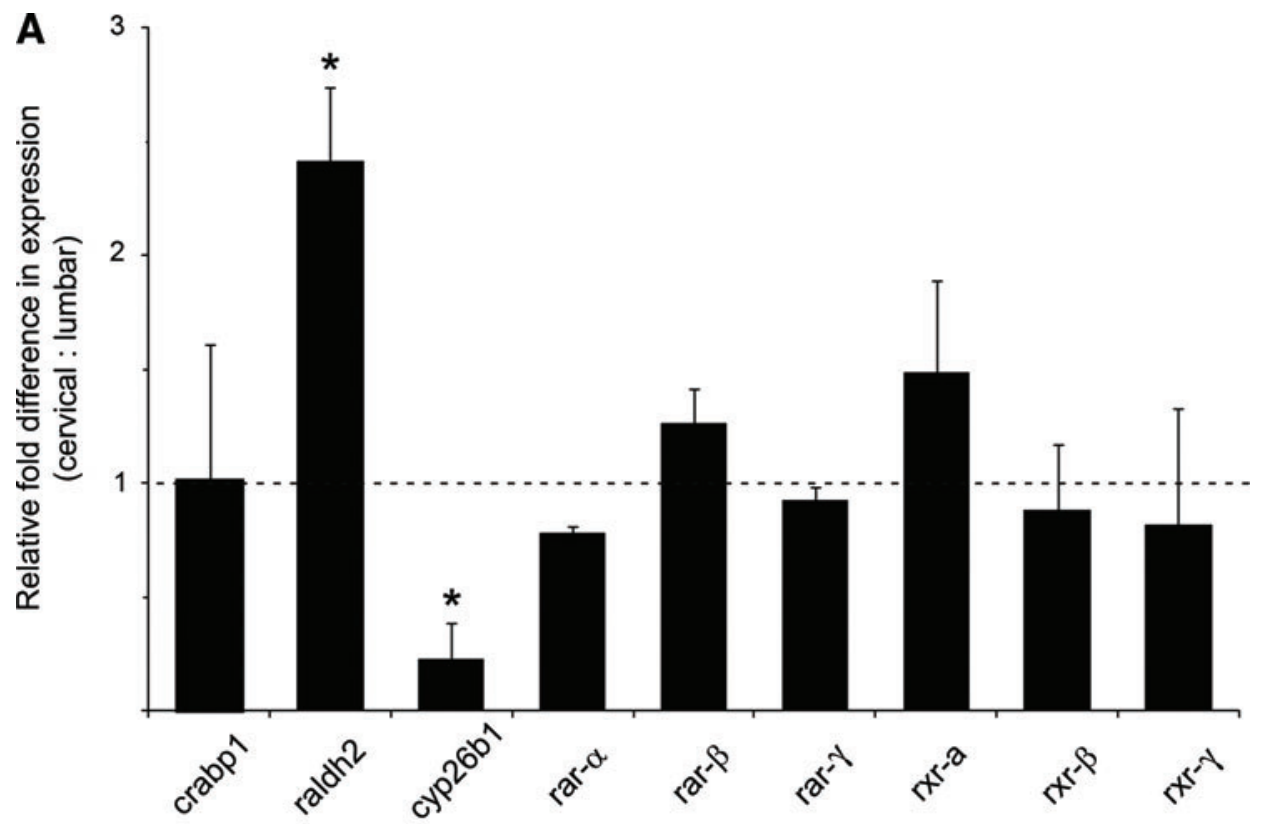

B
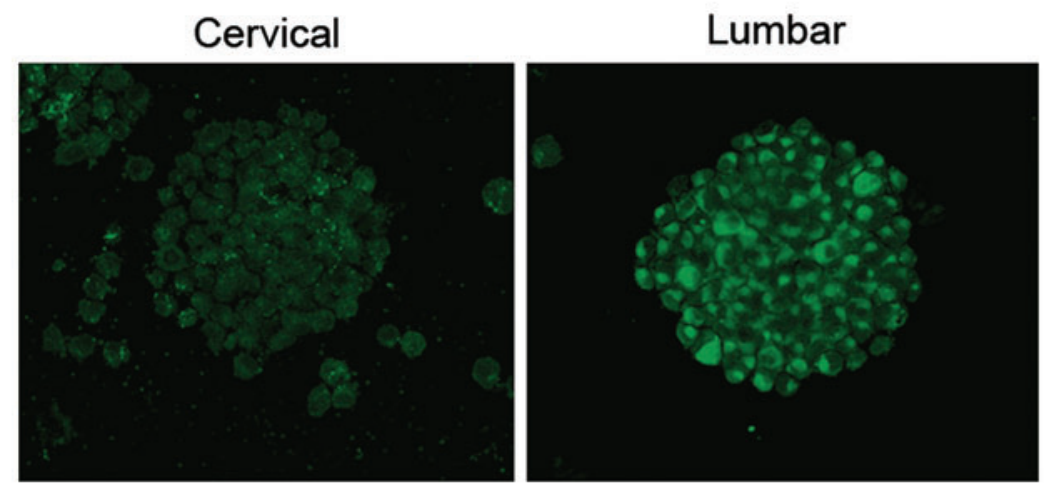

C

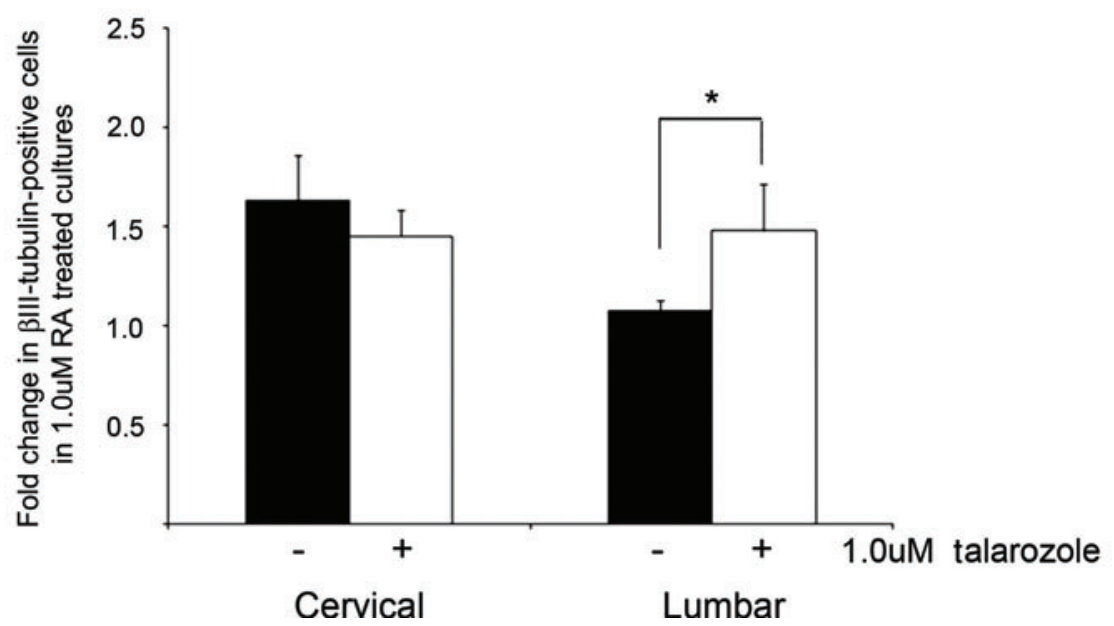

lumbar cells themselves, or whether the neurosphere culture conditions favor the expansion and maintenance of stem cell character of lumbar progenitor cells.

Cells from different axial levels of the developing neural tube are patterned to have different cell-intrinsic regional identities, which specify distinct neuronal subtypes within the region. Embryonic and postnatal neural precursors derived from different brain regions have been shown to retain a region-specific gene expression profile reflecting their region of origin [21-24]. Combinatorial Hox gene expression has also been demonstrated in embryonic spinal cord stem cells [25], but it was unclear whether this was retained in the adult counterpart. We demonstrate here that combinatorial expression of Hox genes is present in progenitor cells derived from the adult spinal cord, indicating that positional identity is preserved. The presence of positional memory in adults 
has also been demonstrated in fibroblasts and mesenchymal cells isolated from different anatomic sites, and more recently in the adult vasculature, where cells are constantly being replenished in the tissue [26-28]. Here we show that positional identity is also present in slowly dividing adult neural progenitor cells from tissue that is not normally selfrenewing. The Hox signatures displayed by the axial progenitor cells are faithfully maintained during multiple passaging, indicating that these cells are intrinsically hard wired to express the genes appropriate to the region from which they were derived. Moreover, the presence of Hox gene expression profiles through multiple passaging suggests an active maintenance of the Hox code during progenitor cell proliferation.

In the postnatal SVZ, neural stem cells from different regions generate different neuronal subtypes [1]. Whether neuronal specification is a result of positional identity genes remains to be determined. In the adult dermis, it was demonstrated that the Hox code can serve as a source of positional memory to regulate region-specific patterning [29]. The importance of positional identity is also underscored in transplant studies, where a mismatch in positional identity between donor and recipient cells is responsible for aberrant bone repair [30]. The expression of Hox genes could convey information necessary for neuronal patterning and functional integration of neurons in the adult spinal cord. Indeed, a recent study demonstrated that ES cell-derived motor neurons, cultured to express a distinct set of Hox genes can differentiate into the corresponding neuronal subtype and innervate their proper targets when transplanted into the chick embryonic spinal cord [31]. Though SCPC-derived neurons are capable of integrating in the neural stem cell niche, specifically, with hippocampal granule neurons after transplantation into the dentate gyrus [14], the inherent Hox code expressed by distinct endogenous SCPC populations could provide an advantage for the derivation and integration of subtype-specific motor neurons during spinal cord repair, and potentially, in muscle target connectivity [32-35]. It would be interesting to determine whether specific motor neuron subtypes could be acquired through the introduction or alteration of Hox code in adult spinal cord progenitor cells. Further studies will be needed to determine which factors are required for the generation of subtype-specific neurons from adult SCPCs.

RA is involved in neural tube patterning, neuronal differentiation, and axon outgrowth [36]. In the adult dentate gyrus, RA continues to play a role during neuronal differentiation to maintain long-term synaptic plasticity [15]. In addition, RA and its precursor retinol have been demonstrated to enhance neurogenesis in adult NSC cultures and explants isolated from the SVZ [16]. However, the role of RA in progenitor cells derived from non-neurogenic zones has not been well studied. Here, we show that RA induces neurogenesis specifically in cervical-derived SCPC cultures, but not in lumbar cells. This increase in neurogenesis occurred through neural progenitor cell proliferation in cervical SCPC cultures, consistent with findings from adult SVZderived neural stem cells indicating a similar function of RA [16]. We found upregulation of Hoxc5 in both RA-treated cervical and lumbar cells, which may reflect the direct regulation of RA on Hoxc5 due to the presence of a conserved RA responsive element (RARE) in the promoter region of
Hoxc5 [37]. It is possible that despite the degradative effects of Cyp26b1 in lumbar-derived cells, not all RA is purged from the cell, and that a minimal concentration of RA contributed to the upregulation observed. It is unclear whether Hoxc5 could play a direct role in enhancing specifically the neurogenicity of cervical progenitors, but neuronal differentiation was unaffected by strong Hoxc5 expression in lumbar progenitors. Nevertheless, the overall Hox gene signature remained unaltered, and cervical progenitors maintained an anterior identity. Expression of posterior genes (Hoxc10, c11, and c13) was unchanged in cervical cells, whereas expression of the anterior Hoxc4 gene was unaltered in lumbar cells. This indicates that in contrast to embryonic stem cells, where positional identity is readily altered by RA [18], there is limited plasticity of the adult Hox code in SCPCs.

We identified that the RA-degrading enzyme Cyp26b1, absent in cervical SCPCs but expressed in lumbar SCPCs, regulates RA signaling in SCPC populations. During embryonic development, Cyp26 enzymes act to regulate RA signaling by degrading and therefore limiting the availability of RA in the cell. In zebrafish hindbrain, Cyp26b1 expression establishes non-neurogenic zones and inhibits neurogenesis by antagonizing RA signaling [38]. During embryonic development, Cyp26a1 is also expressed in the caudal region of the spinal cord and is responsible for protecting the caudal stem cell zone from the differentiating effects of RA. Cyp26b1 is expressed at specific levels of the upper and lower thoracic spinal cord, adjacent to the cervical and lumbar regions that express the RA synthesizing enzyme Raldh2 [39]. Our findings suggest that adult progenitor cells also display differential Cyp26b1 expression and consequently, similar RA signaling activity. We also found high expression of Raldh2 in cervical axial-derived cells. This enzyme acts upstream of RA and therefore is unlikely to contribute to differential signaling pathway activation in our RA-treated cultures. However, it further supports a physiological role for RA signaling in cervical progenitor cells. Conversely, low expression of Raldh2 in lumbar progenitor cells may reflect limited RA signaling in these cell populations. We further demonstrate that RA signaling could be effectively activated in lumbar-derived cultures by blocking Cyp26b1 activity. Inhibition of Cyp26b1 led to a mild but significant increase in neurogenesis in lumbar cultures, suggesting that the combination of RA and Cyp26b1 inhibitors can be a viable approach to stimulate neurogenesis in lumbar regions.

The differential RA responsiveness in spatial progenitor populations suggests that different growth factor regimens may be required for site-specific neuronal regeneration of endogenous SCPCs derived from distinct axial regions. Analogous to the differential RA sensitivity, it is likewise possible that these populations could display differential responsiveness to other niche signals such as FGF or EGF, factors required for the propagation of adult neural stem cell culture, which could also explain the differences in cellular behavior observed for anteroposterior populations. As previous studies on spinal cord progenitor cells were performed on multiple regions of the spinal cord, it would be necessary to reexamine their response to niche factors according to their anatomical origin. Our study implies that progenitor cells can be more efficiently mobilized by identifying the relevant combinations of niche factors. These findings have important implications for treating injury incurred at 
different axial levels, and provide a key step to the development of targeted neuronal replacement therapies based on the recruitment of endogenous cells.

\section{Acknowledgments}

This work was supported by the Liu Po Shan Motor Neuron Disease Endowment Fund and Small Project Funding for Basic Research and the Stem Cell and Regenerative Medicine Consortium of The University of Hong Kong.

\section{Author Disclosure Statement}

No competing financial interests exist.

\section{References}

1. Merkle FT, Z Mirzadeh and A Alvarez-Buylla. (2007). Mosaic organization of neural stem cells in the adult brain. Science 317:381-384.

2. Jessell TM. (2000). Neuronal specification in the spinal cord inductive signals and transcriptional codes. Nat Rev Genet $1: 20-29$.

3. Jung H, J Lacombe, EO Mazzoni, KF Liem, Jr., J Grinstein, S Mahony, D Mukhopadhyay, DK Gifford, RA Young, et al. (2010). Global control of motor neuron topography mediated by the repressive actions of a single hox gene. Neuron 67:781-796.

4. Horner PJ, AE Power, G Kempermann, HG Kuhn, TD Palmer, J Winkler, LJ Thal and FH Gage. (2000). Proliferation and differentiation of progenitor cells throughout the intact adult rat spinal cord. J Neurosci 20:2218-2228.

5. Yamamoto S, N Yamamoto, T Kitamura, K Nakamura and M Nakafuku. (2001). Proliferation of parenchymal neural progenitors in response to injury in the adult rat spinal cord. Exp Neurol 172:115-127.

6. Kulbatski I, AJ Mothe, A Keating, Y Hakamata, E Kobayashi and CH Tator. (2007). Oligodendrocytes and radial glia derived from adult rat spinal cord progenitors: morphological and immunocytochemical characterization. J Histochem Cytochem 55:209-222.

7. Meletis K, F Barnabe-Heider, M Carlen, E Evergren, N Tomilin, O Shupliakov and J Frisen. (2008). Spinal cord injury reveals multilineage differentiation of ependymal cells. PLoS Biol 6:e182.

8. Horky LL, F Galimi, FH Gage and PJ Horner. (2006). Fate of endogenous stem/progenitor cells following spinal cord injury. J Comp Neurol 498:525-538.

9. Mothe AJ, I Kulbatski, A Parr, M Mohareb and CH Tator. (2008). Adult spinal cord stem/progenitor cells transplanted as neurospheres preferentially differentiate into oligodendrocytes in the adult rat spinal cord. Cell Transplant 17:735-751.

10. Johansson CB, M Svensson, L Wallstedt, AM Janson and J Frisen. (1999). Neural stem cells in the adult human brain. Exp Cell Res 253:733-736.

11. Namiki J and CH Tator. (1999). Cell proliferation and nestin expression in the ependyma of the adult rat spinal cord after injury. J Neuropathol Exp Neurol 58:489-498.

12. Weiss S, C Dunne, J Hewson, C Wohl, M Wheatley, AC Peterson and BA Reynolds. (1996). Multipotent CNS stem cells are present in the adult mammalian spinal cord and ventricular neuroaxis. J Neurosci 16:7599-7609.

13. Shihabuddin LS, J Ray and FH Gage. (1997). FGF-2 is sufficient to isolate progenitors found in the adult mammalian spinal cord. Exp Neurol 148:577-586.
14. Shihabuddin LS, PJ Horner, J Ray and FH Gage. (2000). Adult spinal cord stem cells generate neurons after transplantation in the adult dentate gyrus. J Neurosci 20:8727-8735.

15. Jacobs S, DC Lie, KL DeCicco, Y Shi, LM DeLuca, FH Gage and RM Evans. (2006). Retinoic acid is required early during adult neurogenesis in the dentate gyrus. Proc Natl Acad Sci U S A 103:3902-3907.

16. Wang TW, H Zhang and JM Parent. (2005). Retinoic acid regulates postnatal neurogenesis in the murine subventricular zone-olfactory bulb pathway. Development 132:2721-2732.

17. Wichterle H, I Lieberam, JA Porter and TM Jessell. (2002). Directed differentiation of embryonic stem cells into motor neurons. Cell 110:385-397.

18. Okada Y, T Shimazaki, G Sobue and H Okano. (2004). Retinoic-acid-concentration-dependent acquisition of neural cell identity during in vitro differentiation of mouse embryonic stem cells. Dev Biol 275:124-142.

19. Stoppie P, M Borgers, P Borghgraef, L Dillen, J Goossens, G Sanz, H Szel, C Van Hove, G Van Nyen, et al. (2000). R115866 inhibits all-trans-retinoic acid metabolism and exerts retinoidal effects in rodents. J Pharmacol Exp Ther 293:304-312.

20. Geada AM, SJ Gaunt, M Azzawi, SM Shimeld, J Pearce and PT Sharpe. (1992). Sequence and embryonic expression of the murine Hox-3.5 gene. Development 116:497-506.

21. Parmar M, C Skogh, A Bjorklund and K Campbell. (2002). Regional specification of neurosphere cultures derived from subregions of the embryonic telencephalon. Mol Cell Neurosci 21:645-656.

22. Seaberg RM, SR Smukler and D van der Kooy. (2005). Intrinsic differences distinguish transiently neurogenic progenitors from neural stem cells in the early postnatal brain. Dev Biol 278:71-85.

23. Hitoshi S, V Tropepe, M Ekker and D van der Kooy. (2002). Neural stem cell lineages are regionally specified, but not committed, within distinct compartments of the developing brain. Development 129:233-244.

24. Onorati M, M Binetti, L Conti, S Camnasio, G Calabrese, I Albieri, F Di Febo, M Toselli, G Biella, et al. Preservation of positional identity in fetus-derived neural stem (NS) cells from different mouse central nervous system compartments. Cell Mol Life Sci 68:1769-1783.

25. Kelly TK, SL Karsten, DH Geschwind and HI Kornblum. (2009). Cell lineage and regional identity of cultured spinal cord neural stem cells and comparison to brain-derived neural stem cells. PLoS One 4:e4213.

26. Chang HY, JT Chi, S Dudoit, C Bondre, M van de Rijn, D Botstein and PO Brown. (2002). Diversity, topographic differentiation, and positional memory in human fibroblasts. Proc Natl Acad Sci U S A 99:12877-12882.

27. Ackema KB and J Charite. (2008). Mesenchymal stem cells from different organs are characterized by distinct topographic Hox codes. Stem Cells Dev 17:979-991.

28. Pruett ND, RP Visconti, DF Jacobs, D Scholz, T McQuinn, JP Sundberg and A Awgulewitsch. (2008). Evidence for Hoxspecified positional identities in adult vasculature. BMC Dev Biol 8:93.

29. Rinn JL, JK Wang, N Allen, SA Brugmann, AJ Mikels, H Liu, TW Ridky, HS Stadler, R Nusse, JA Helms and HY Chang. (2008). A dermal HOX transcriptional program regulates site-specific epidermal fate. Genes Dev 22:303-307.

30. Leucht P, JB Kim, R Amasha, AW James, S Girod and JA Helms. (2008). Embryonic origin and Hox status determine progenitor cell fate during adult bone regeneration. Development 135:2845-2854. 
31. Peljto M, JS Dasen, EO Mazzoni, TM Jessell and H Wichterle. Functional diversity of ESC-derived motor neuron subtypes revealed through intraspinal transplantation. Cell Stem Cell 7:355-366.

32. Dasen JS, JP Liu and TM Jessell. (2003). Motor neuron columnar fate imposed by sequential phases of Hox-c activity. Nature 425:926-933.

33. Dasen JS, BC Tice, S Brenner-Morton and TM Jessell. (2005). A Hox regulatory network establishes motor neuron pool identity and target-muscle connectivity. Cell 123:477-491.

34. Novitch BG, AI Chen and TM Jessell. (2001). Coordinate regulation of motor neuron subtype identity and pan-neuronal properties by the bHLH repressor Olig2. Neuron 31:773-789.

35. Sockanathan S and TM Jessell. (1998). Motor neuron-derived retinoid signaling specifies the subtype identity of spinal motor neurons. Cell 94:503-514.

36. Maden M. (2007). Retinoic acid in the development, regeneration and maintenance of the nervous system. Nat Rev Neurosci 8:755-765.

37. Arcioni L, A Simeone, S Guazzi, V Zappavigna, E Boncinelli and F Mavilio. (1992). The upstream region of the human homeobox gene HOX3D is a target for regulation by retinoic acid and HOX homeoproteins. EMBO J 11:265-277.
38. Gonzalez-Quevedo R, Y Lee, KD Poss and DG Wilkinson. Neuronal regulation of the spatial patterning of neurogenesis. Dev Cell 18:136-147.

39. Abu-Abed S, G MacLean, V Fraulob, P Chambon, M Petkovich and P Dolle. (2002). Differential expression of the retinoic acid-metabolizing enzymes CYP26A1 and CYP26B1 during murine organogenesis. Mech Dev 110:173-177.

Address correspondence to: Mai Har Sham, Ph.D. Department of Biochemistry The University of Hong Kong Li Ka Shing Faculty of Medicine Building, 3/F Laboratory Block 21 Sassoon Road, Pokfulam Hong Kong SAR China

E-mail: mhsham@hku.hk

Received for publication October 30, 2011 Accepted after revision December 31, 2011 Prepublished on Liebert Instant Online January 4, 2012 